\title{
Numerical Study of Quench Protection for Fast-Ramping Accelerator Magnets
}

\author{
Nikolai Schwerg, Bernhard Auchmann, Karl-Hubert Mess, and Stephan Russenschuck
}

\begin{abstract}
The quench module of the ROXIE field computation program has been presented at previous conferences. In this paper we discuss recently implemented features that allow quench simulation of fast-ramping superconducting magnets. As the reliability of quench detection during the ramps depends on the signal to noise ratio, we simulate the influence of detection thresholds and the propagation of undetected quenches during the ramps. We also study the effect of an increased copper content and the feasibility of a self-protected magnet surviving a powering cycle with an undetected quench and without quench heater firing or energy-extraction system.
\end{abstract}

Index Terms-Fast-ramping magnets, quench simulation.

\section{INTRODUCTION}

$\mathbf{T}$ HE quench protection of superconducting accelerators is usually based on a reliable detection of a quench, the decoupling of the quenching magnet from the non-quenching magnets by means of diodes or thyristors, an energy extraction system, and the shut-down of the power converters. In recent projects, e.g., the SIS300 dipole magnet for the FAIR project [1], ramp-rates of $1 \mathrm{~T} / \mathrm{s}$, i.e. about $1100 \mathrm{~A} / \mathrm{s}$, are proposed. The flat-top is considered to last from 10 to 100 seconds. Considering an inductance of about $25 \mathrm{mH}$ for a magnet length of $2.9 \mathrm{~m}$, the ramp-induced voltage across a magnet is $27.5 \mathrm{~V}$. The ring will be powered by several power converters in order to deal with the total voltage of e.g., $3000 \mathrm{~V}$ for an installation of 110 magnets.

Calculations in Section VI show that a quench detection threshold of $1 \mathrm{~V}$ will be desirable during the ramp in order to protect the magnets. The precision of the electronics must be at least a factor 10 better in order to cover unexpected behavior such as parasitic transient effects. This is no technological challenge at the flat-top. However, during the ramp the induced voltage across a magnet rises in a short time to $27.5 \mathrm{~V}$, while the common mode voltage rises to $\pm 1500 \mathrm{~V}$ (or $300 \mathrm{~V}$ if five independent powering sub-sectors are chosen). In particular during the acceleration and deceleration of the ramps, the required $50 \mathrm{~dB}$ signal to noise ratio (detection precision of $0.1 \mathrm{~V}$ over a signal of $27.5 \mathrm{~V}$ ) "riding" on a rapidly changing $84 \mathrm{~dB}$ common mode background (Ratio of precision over

Manuscript received August 22, 2008. First published June 23, 2009; current version published July $15,2009$.

The authors are with CERN AT, CH-1211 Geneva 23, Switzerland (e-mail: nikolai.schwerg@cern.ch; bernhard.auchmann@cern.ch; karl.hubert. mess@cern.ch; stephan.russenschuck@cern.ch).

Color versions of one or more of the figures in this paper are available online at http://ieeexplore.ieee.org.

Digital Object Identifier 10.1109/TASC.2009.2018790 common mode voltage) is a challenge in a large installation where reliability is the most important requirement.

Based on the fact that a magnet designed for fast-ramping operation can be ramped down much faster than existing superconducting magnets, an alternative possibility can be considered. It would be sufficient to detect a quench on the flat-top or at the injection plateau, if the magnets can survive an undetected quench during the ramp. Bypass diodes would not be needed in this case, which themselves represent a technological challenge. If state-of-the art power converters with capacitive storage are used, even the dump resistors and switches would not be required.

We give a brief overview of the recent extensions to the ROXIE quench module. Section III gives details on the model magnet and the protection system assumed for our simulations. In the subsequent sections we discuss four aspects of quench simulation for fast-ramping magnets:

- In Section IV we show the influence of different thermal models (see companion paper [2]) on the simulated coil temperature during the powering cycle.

- In Section V we study the ramp-rate dependence of the quench current.

- In Section VI we investigate the peak temperature in a magnet for undetected quenches during the ramp phases. We compare the cases of quench detection during ramp for a threshold of $1 \mathrm{~V}$ and the $100 \mathrm{mV}$ threshold that is standard in slowly-ramping magnets.

- In Section VII we discussed whether additional coppercontent in the coil could protect a fast-ramping magnet for an undetected quench during the ramps, or even during an entire cycle.

The authors emphasize that this paper is not meant as a quench protection study for the SIS300 dipole, as the results strongly depend on the material parameters, e.g., of the electrical insulation, and the protection scheme. We focus on the recently implemented features of the ROXIE quench module, which enable us to simulate quench phenomena in fast-ramping magnets.

\section{QUENCH MODEL FOR FAST-RAMPING MAGNETS}

A quench is the resistive transition of a superconductor that occurs if the current density, the magnetic field in the cable, or the cable temperature exceeds a critical value. From this description it is evident that quench simulation requires a multiphysics modeling approach. A detailed description of the numerical models is given in [3] and in the companion paper [2].

The ROXIE framework [4] allows to accurately model the interdependence of various physical effects. Iteration schemes and adaptive time-stepping are implemented to resolve the highly 
TABLE I

DATA USED FOR QUENCH SIMULATIONS BASED ON THE SIS300 DIPOLE MAGNET AS DEFINED IN [5]

\begin{tabular}{l|l}
\hline Quantity & Value \\
\hline \hline Length & $1 \mathrm{~m}$ \\
Magnetic length & $0.75 \mathrm{~m}$ \\
Ramp rate & $1 \mathrm{~T} / \mathrm{s}$ \\
Differential inductance & $7.5-9.7 \mathrm{mH}$ \\
\hline \hline Detection threshold & $0.1-1 \mathrm{~V}$ \\
Quench heaters delay & about $90 \mathrm{~ms}$ \\
Quench heaters covering & 20 conductors per \\
& quadrant in outer layer \\
Dump resistor & $200 \mathrm{~m} \Omega$ \\
Dump resistor switch delay & $50 \mathrm{~ms}$ \\
Cold diode forward voltage & $6 \mathrm{~V}$ \\
\hline \hline RRR & 278 \\
copper-to-superconductor ratio & 1.38 \\
Contact resistance & $20 \mathrm{~m} \Omega$ \\
Adjacent resistance & $200 \mu \Omega$ \\
Operation Temperature & $4.7 \mathrm{~K}$ \\
Percentage of Helium in cable area & $10 \%$ \\
\hline
\end{tabular}

nonlinear behaviour of materials such as helium at cryogenic temperatures. For quench simulation, the user is required to supply data for the characterization of quench-heater systems, protection circuits and electronics, electrical and thermal properties of compound cable materials and insulators, geometrical and magnetic data of the magnets, and many more parameters. The data must be available at different cryogenic temperatures, and for a range of fields and pressures.

Given the large number of empirical parameters in the models, it must be noted that a important part of the simulation work consists in the determination of parameters such that the simulation matches the measurement. It is important to realize that only when

- all relevant phenomena have been taken into account and modelled accurately,

- the choice of material parameters is physically reasonable and within the given range of uncertainty of measured values,

- the simulation result matches the measured data within the uncertainty of the measurement,

we are able to reproduce the internal states of a quenching magnet, i.e., observe quantities that evade measurement such as hot spot temperature and internal voltages.

\section{The Model Magnet}

For the purpose of this paper we simulate the 2-layer design of a SIS300 dipole magnet as defined in [5]. In Table I we summarize the relevant data for the quench calculation. The non linear differential inductance is given by $L_{\mathrm{d}}(I)=\partial \Psi / \partial I$.

We consider conductive cooling via the Kapton insulation to the helium bath across the inner and outer radial surfaces of the coil. The ramp cycle is given in [5] as an up-ramp from $1.6 \mathrm{~T}$ to $6 \mathrm{~T}$ in $4.4 \mathrm{~s}$, followed by a plateau at $6 \mathrm{~T}$ of $11 \mathrm{~s}$, and a down ramp to $1.6 \mathrm{~T}$ in $4.4 \mathrm{~s}$.

\section{COOLING SCHEMES}

The temperature variation during a ramp-cycle can be calculated with three different thermal diffusion models:

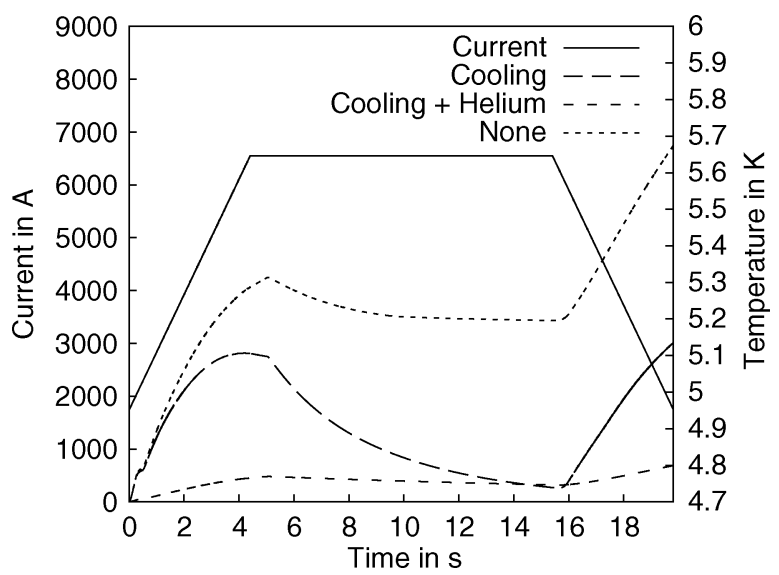

Fig. 1. Temperature variation during ramp_cycle of the SIS300 dipole magnet.

1) Adiabatic conditions, i.e., no cooling and no helium as it is appropriate for potted coils.

2) Heat transfer across the inner and outer radial surfaces without considering confined helium inside the cable.

3) Modeling of conductive cooling (see above) and of the thermal capacity of the confined helium (about 10\%) inside the cable.

Fig. 1 shows the temperature variation during one ramp-cycle for the three cases. The temperature variation per cycle is an order of magnitude smaller for the wetted coil, as the heat capacity of helium is dominant at low temperatures. In all cases the temperature decreases at the flat-top. In case of the adiabatic model 1) this is due to the longitudinal thermal conduction, which is, however, not sufficient to prevent a quench already in the second cycle. In this paper we use the model 3, which reproduces data published in [5].

\section{QUENCH LIMITS AT DIFFERENT RAMP RATES}

In Fig. 2 we see the evolution of current and peak-temperature inside the magnet for quenches near the flat-top. The magnet is ramped until the quench limit is reached and the quench protection system detects a resistive voltage of $100 \mathrm{mV}$. The magnet is protected with quench heaters and a dump resistor, see Table I. The ramp rate varies between $1.0 \mathrm{~T} / \mathrm{s}$ and $4.5 \mathrm{~T} / \mathrm{s}$.

We observe how the temperature in the magnet rises due to ramp-induced losses [6] that reduce the temperature margin to quench. The quench current thus decreases for higher ramp rates. The peak temperature after quench is also reduced as a consequence of the lower quench current.

In Fig. 3 the temperature margin to quench is shown for each conductor in the cross-section as a function of time. The plot corresponds to the fastest ramp-rate in Fig. 2, i.e. a ramp-rate of $4.5 \mathrm{~T} / \mathrm{s}$. The margin reduces steadily as a consequence of increasing current and field, and due to induced losses. At $t=$ $1.18 \mathrm{~s}$ the magnet quenches in block number 5, see Fig. 7. The quench is detected at $t=1.19 \mathrm{~s}$ and the quench heaters are fired at $t=1.24 \mathrm{~s}$. At the same time the dump resistor is switched into the circuit and the current drops sharply. The quench heaters are effective about $40 \mathrm{~ms}$ later.

The temperature margin in the inner layer coil increases after the quench. As a matter of fact, a number of conductors recover 


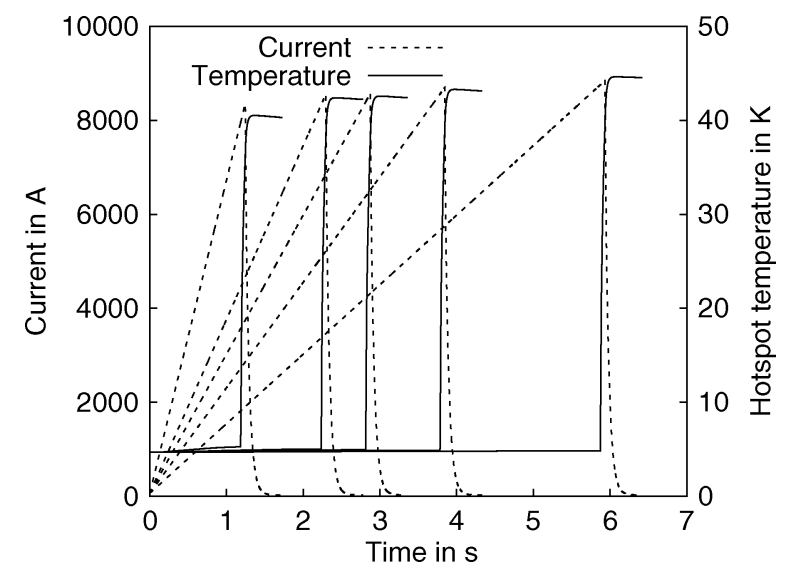

Fig. 2. Current and peak temperature in a magnet during an up-ramp to the quench limit and quench detection. The graphs correspond to ramp rates (from right to left) of $1.0 \mathrm{~T} / \mathrm{s}, 1.5 \mathrm{~T} / \mathrm{s}, 2.0 \mathrm{~T} / \mathrm{s}, 2.5 \mathrm{~T} / \mathrm{s}$, and $4.5 \mathrm{~T} / \mathrm{s}$.

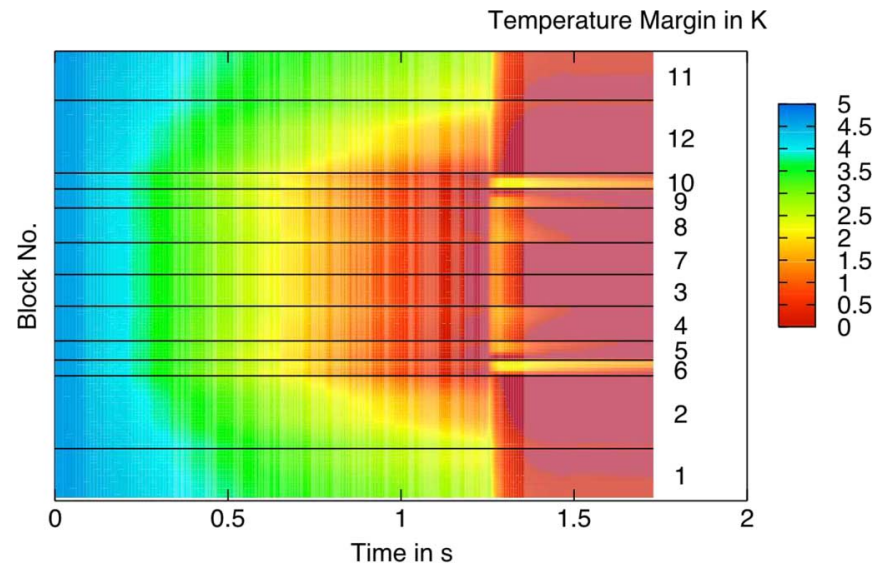

Fig. 3. Temperature margin to quench versus time for an up-ramp with $4.5 \mathrm{~T} / \mathrm{s}$. Block $1,2,11$ and 12 belong to the outer layer and are partially covered by quench heaters.

from quench. This is explained by the fact that the current density and field in the superconductor drops quickly. The time constant of the induced eddy-current losses in the cable, however, is of about $50 \mathrm{~ms}$. Moreover, the heat capacity of the confined helium in the cable results in a long thermal time constant. This explains why the temperature rise due to induced losses is slow and the margin grows immediately after the dump resistor is switched in. Eventually the inner layer is quenched by induced losses. The block number 6, see Fig. 7, does not get an equal share of induced losses. The reason is that it is placed in parallel to the electromagnetic field. The Rutherford cable used for the SIS300 magnet has a stainless steel core, which reduces the cross-over resistance. Consequently, very little eddy-currents are induced in a cable that is positioned in parallel to the field lines.

\section{QUENCh DETECTION DuRING THE UP- AND DOWn RAMP}

We study the current decay and peak-temperature after a quench in the up-ramp. Different detection scenarios are investigated. Quenches are assumed to occur at either 50\%, $75 \%$, or $90 \%$ of the plateau level. Quenches are detected either only at the plateau, or when the resistive voltage has reached

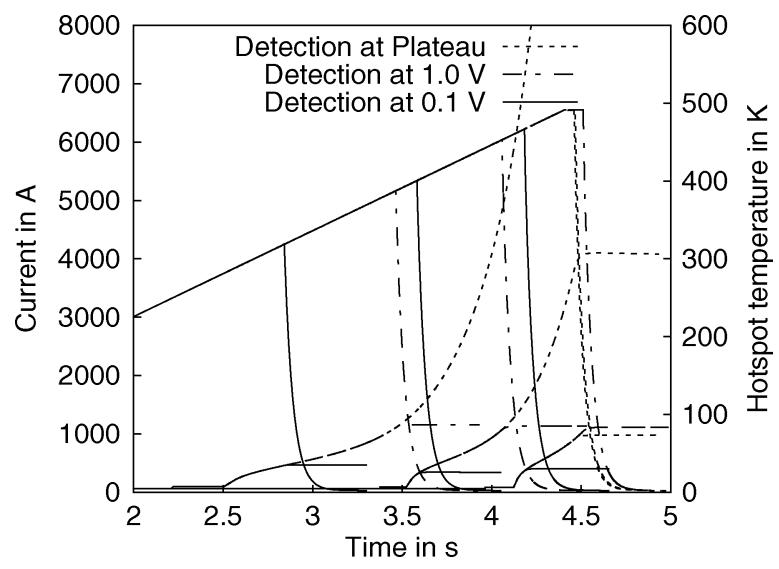

Fig. 4. Current decay and peak-temperature for quenches during the up-ramp phase. Quenches are initiated at $50 \%(2.2 \mathrm{~s}), 75 \%$, or $90 \%$ of the nominal current level. It is assumed that quenches can only be detected at the plateau (dashed line), at a resistive voltage threshold of $1 \mathrm{~V}$ (dash-dotted line), or $100 \mathrm{mV}$ (continuous line).

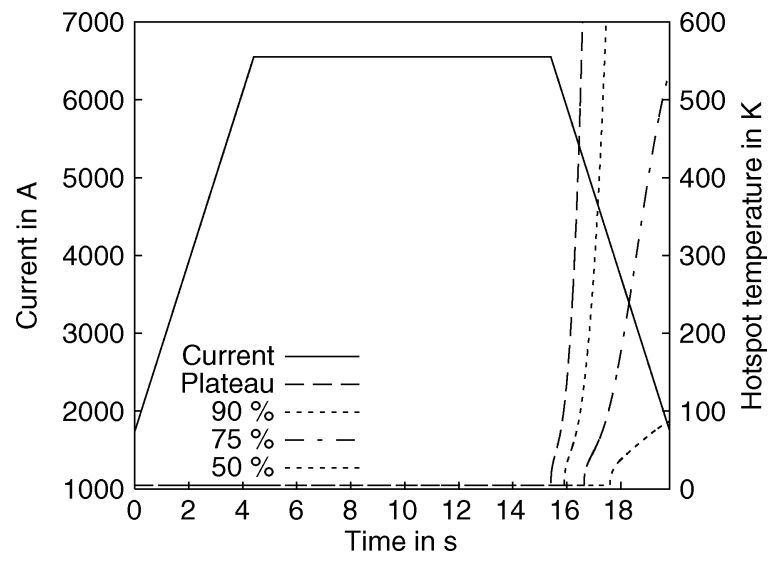

Fig. 5. Current- and peak-temperature evolution for quenches during the downramp. Quenches start at the end of the plateau, at $90 \%, 75 \%$, and $50 \%$ of the nominal current level. It is assumed that quenches can only be detected at the injection level. Comparison with the dashed lines in Fig. 4 shows that the quenches during the down-ramp are more critical than during the up-ramp.

a threshold of $100 \mathrm{mV}$ or $1 \mathrm{~V}$. Fig. 4 shows the current and peak-temperature evolution for the nine different cases.

It can be seen that an undetected quench during the up-ramp leads to a fatal temperature rise in the magnet. The earlier the quench occurs during the up-ramp phase, the higher is the peaktemperature. It turns out that a detection threshold of $1 \mathrm{~V}$ during the ramp is sufficient to protect the magnet against quenches occurring at $50 \%$ of the plateau. The delays of the protection system can be deduced from Fig. 4 by taking the time laps between the first rise in temperature and the point where the current decays and the energy is extracted by the dump resistor. The protection delays are longer at lower excitation, since the resistive voltage rises more slowly.

In Fig. 5 the same exercise is carried out for quenches occurring on the down-ramp. It is assumed that quenches can only be detected at the injection level. Comparison with Fig. 4 shows that quenches occurring near the upper plateau are more critical during the down-ramp than during the up-ramp as it must be expected, since a current is forced to flow through the quenched magnet during a longer period of time. 


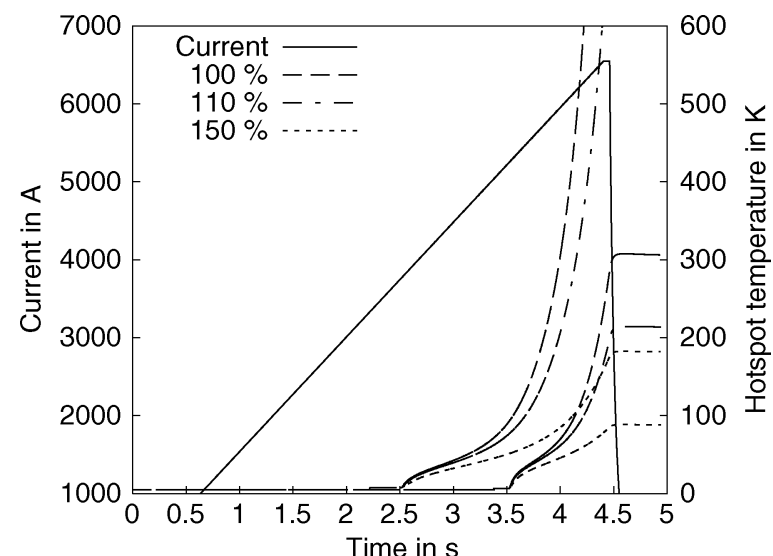

Fig. 6. Peak-temperature and current evolution during quenches at $50 \%$ and $75 \%$ of the plateau level. It is assumed that the quench cannot be detected until the plateau is reached. The simulation is carried out for the SIS300 conductor, as well as for conductors that have a $10 \%$ to $50 \%$ higher copper content.
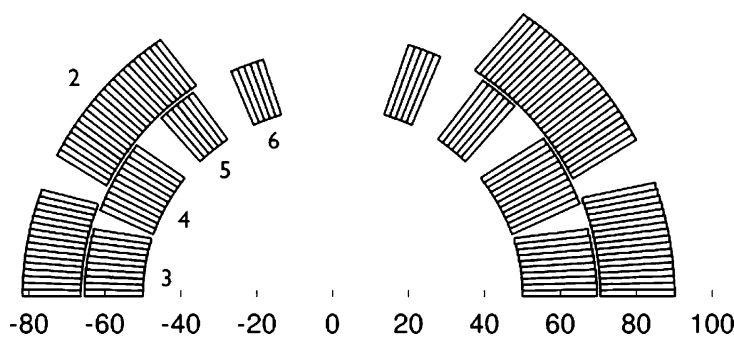

Fig. 7. Left: The standard cross-section of the SIS300 two-layer dipole. Right: The modified cross-section with a wider cable and additional strands for a $50 \%$ increase in copper content. In order to keep the main field, the field quality and the quench margin unchanged, two conductors had to be added to the outer layer. The axis shows the $\mathrm{x}$-position in $\mathrm{mm}$.

\section{VII. "Self-Protecting” Magnets}

We can also investigate how much copper would need to be added to the Rutherford-type cable so that the magnet survives an undetected quench during a ramp. Fig. 6 shows quenches occurring at $50 \%$ and at $75 \%$ of the plateau for different copper content. The baseline of $100 \%$ is given by the cable described in Table I. In case of $150 \%$ copper we added strands and increased the copper-to-superconductor ratio for all strands, to result in 50\% more copper and the same amount of $\mathrm{Nb}$-Ti superconductor. As the cables become wider, the coil cross-section is re-designed with two more conductors in the outer layer, in order maintain the field quality, Fig. 7. The inductance of this coil increases by about 10\%. It can be seen in Fig. 6 that this magnet could survive a quench that occurs half-way on the up-ramp and that is detected only at the plateau.

Protection may be reduced to a strict minimum, if a magnet contains enough copper to survive a full powering cycle with an undetected quench. We assume that a quench occurs at $50 \%$ of the up-ramp, that it is detected at the plateau, and that the magnet is then ramped-down immediately with the regular ramp rate. For the cable with $50 \%$ extra copper, Fig. 8 shows the temperature evolution. This cable had survived an undetected quench during an up ramp, see Fig. 6. We can see that the magnet is

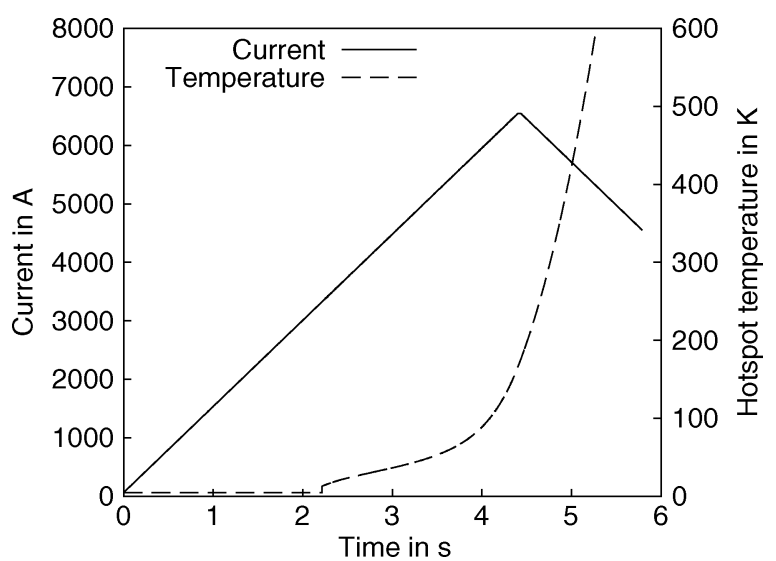

Fig. 8. Peak temperature and current evolution during a quench. The quench starts at $50 \%$ of the up-ramp. It is detected at the plateau. Then the magnet is immediately driven down with the standard slope.

not sufficiently protected for the shortened cycle. It follows that for a magnet with only $50 \%$ additional copper a method of fast current shut-down is indispensable.

\section{CONCLUSION}

With the recently implemented quench-simulation features in the CERN field computation ROXIE, it is possible to study protection schemes for fast ramping magnets. We have carried out qualitative studies of the effect of detection thresholds, cable design parameters, and protection schemes. The quantitative estimate of the peak temperature during the quench requires the gauging of the empirical input data (copper RRR, heat transfer coefficients, quench heater efficiency etc.) with the measurements on a model magnet. In the case studied in Section VII we can show that an undetected quench during a ramp is only tolerable with an increased copper content in the conductors. Otherwise a threshold voltage of $1 \mathrm{~V}$ during the ramp phase is enough to protect the magnet. We have also seen that the model magnet with additional copper cannot be protected without a method of fast current shut-down.

\section{REFERENCES}

[1] W. Henning, "Fair-An international accelerator facility for research with ions and antiprotons," in Proceedings of EPAC 2004, Nov. 2004, pp. 50-53.

[2] N. Schwerg, B. Auchmann, and S. Russenschuck, "Challenges in the thermal modelling of quenches with ROXIE," IEEE Trans. Appl. Supercond., vol. 19, no. 3, June 2009, submitted for publication.

[3] N. Schwerg, B. Auchmann, and S. Russenschuck, "Quench simulation in an integrated design environment for superconducting magnets," IEEE Trans. Magn., vol. 44, no. 6, pp. 934-937, June 2008.

[4] "ROXIE: Routine for the optimization of magnet X-sections, inverse field calculation and coil end design," in Proceedings of the First international ROXIE Users Meeting and Workshop, S. Russenschuck, Ed., Geneva, Switzerland, March 1998.

[5] S. Kozub, I. Bogdanov, A. Seletsky, P. Shcherbakov, V. Sytnik, L. Tkachenko, and V. Zubko, Final Report on the Research and Development Contract "Technical Design of the sis-300 dipole model" IHEP, Protvino, July 2006, Tech. Rep..

[6] A. Verweij, "Electrodynamics of Superconducting Cables in Accelerator Magnets," Ph.D. dissertation, Universiteit Twente, Enschede, Netherlands, 1995. 